\title{
Improving Tracking Accuracy of a MIMO State Feedback Controller for Elastic Joint Robots
}

\author{
Luc Le-Tien and Alin Albu-Schäffer
}

\begin{abstract}
In this paper a control scheme is addressed to improve the tracking accuracy of flexible joint robots without replacing the structure of a MIMO state feedback controller which is used effectually with the DLR medical robots. By using the desired position, the new desired link torque, as well as their derivatives the effects of nonlinear dynamics are compensated and the tracking accuracy is thereby increased. Hereby, the new desired link torque takes the whole rigid body dynamics into account, not only the friction and gravitation compensation terms. A stability analysis based on the Lyapunov theory and Barbalat's lemma is given for this new MIMO state feedback control scheme. Experimental results validate the practical efficiency of the approach.
\end{abstract}

Index Terms-State feedback control, decoupling control, flexible joint robots

\section{INTRODUCTION}

The DLR medical robots have been developed for a variety of specific medical applications. They are flexible joint robots with high gear ratio aiming at low own weight and high payload. For several applications of DLR medical robots, such as navigated pedicle screw placement, biopsies [1], laser cutting or minimally invasive surgery [2] a high positioning accuracy is required.

In Fig. 1 the second generation of the DLR medical robots is shown, the so-called MiroSurge, which is specialized for minimally invasive surgery. Typical for these robots is the existence of pairs of joints which are highly coupled. In addition, the joints have a high compliance.

Path tracking errors mainly originate from kinematic errors, control performance limitations, and joint flexibility. The kinematic errors can easily be compensated in the path planning. So the control performance and flexibility of the flexible joint robot must be taken into account to achieve small path tracking errors.

For elastic joint robots different control strategies have been developed in the literature, such as PD [3], SISO state feedback [4], feedback linearization [5], backstepping [6], or passivity based adaptive control [6], [7]. Because of the robustness only the controllers PD and SISO state feedback are used in practice for the DLR medical robots, but with miserable results for the coupled joints.

In order to deal with the strong joint coupling of the DLR medical robot, in [8] a MIMO state feedback controller with full state feedback (motor position, link side torque, as well as their derivatives), gravity and friction compensation was

Luc Le-Tien is with The Robotics and Mechatronics Center, German Aerospace Center (DLR), Oberpfaffenhofen, D-82234 Wessling, Germany Luc.Le-Tien@dlr.de

Alin Albu-Schäffer is with The Robotics and Mechatronics Center, German Aerospace Center (DLR), Oberpfaffenhofen, D-82234 Wessling, Germany Alin.Albu-Schaefferdalr.de introduced. This controller has already been successfully applied to the DLR medical robot and is mostly used in medical applications as the standard position controller. However, this controller does not take all effects into account, e.g. not the full motor dynamics, nonlinear link dynamics (e.g. centrifugal or coriolis forces), and especially tracking motor velocity, which result in deviations of the robot motion. So the inclusion of these nonlinear effects is really necessary to improve the path tracking accuracy.

In motion control, position deviations can be clearly reduced by friction compensation. There exists a huge amount of the approaches to friction compensation, such as model based friction compensation [9], [10], adaptive friction compensation [11], integrators or disturbance observers [12]. But this is not the subject of this work. In this paper a change of the MIMO state feedback controller with model based friction compensation in [8] is addressed to increase the tracking accuracy of the DLR medical robots without replacing its structure. Therefor, the desired motor position and the desired link torque are newly calculated by using the whole rigid body dynamics, not only the gravitation as in the old control structure. Thereby, the compensation of the nonlinear dynamics considers the link velocity error for the computation of the desired motor position and the desired link torque to ensure the system stability. Furthermore, the desired motor velocity and the derivative of the new desired link torque are added to the controller to improve the tracking accuracy.

In this paper the joint damping is neglected for control design, as well as for stability analysis. But experiments are shown that the neglect of the joint damping in this new control structure hardly ever changes the system performance.

The system in this case is non-autonomous, so the stability of system can be proven by using the Barbalat's lemma instead of the Krasovskii-LaSalle principle as in [8].

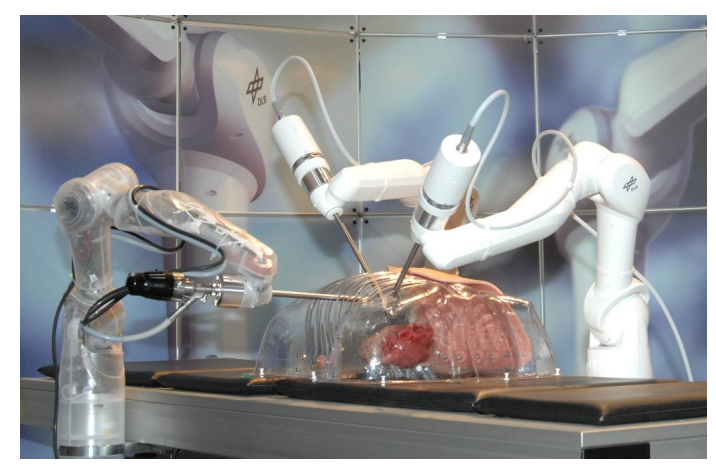

Fig. 1. The DLR MiroSurge telerobotic system for endoscopic surgery. 
Because the friction compensation plays a role for the tracking position, in practice this new control structure can be combined with different friction compensation methods to achieve a higher accuracy (e.g. disturbance observer [12]).

The paper is organized as follows. Section II introduces the dynamic robot model. In Sec. III the standard structure of a coupling MIMO state feedback controller without feedforward for the DLR medical robots is recapitulated. In Sec. IV the new controller with feedforward is presented and its stability is analyzed. Finally, the obtained performance is verified by experimental tests reported in Sec. V.

\section{MODEL OF THE ROBOT DYNAMICS}

The DLR medical robot (in Fig. 1) has $n=7$ rotary joints that exhibit considerable elasticity. Apart from the first joint, the following three joint pairs are coupled with differential gears. The simplified dynamics of the DLR medical robot with flexible joints [8] is described by

$$
\begin{aligned}
u_{m} & =J_{m} \ddot{\theta}_{m}+T^{T} \tau+\tau_{f m} \\
\tau & =M(q) \ddot{q}+C(q, \dot{q}) \dot{q}+g(q) .
\end{aligned}
$$

Therein, $q \in \mathbb{R}^{n}$ and $\theta_{m} \in \mathbb{R}^{n}$ are the link and motor angles, respectively. $\tau_{f m} \in \mathbb{R}^{n}$ is the friction moment. The control input is the motor torque $u_{m} \in \mathbb{R}^{n}$. The motor inertia matrix $J_{m} \in \mathbb{R}^{n \times n}$ is diagonal and positive definite. $\mathrm{T}$ is the transformation matrix of the differential gear. The transmission torque between motor and link dynamics $\tau \in$ $\mathbb{R}^{n}$ is modeled as a linear function of the motor and the link position

$$
\tau=K\left(T \theta_{m}-q\right)
$$

and is measured by strain gauge based torque sensors. The joint stiffness matrix $K \in \mathbb{R}^{n \times n}$ is symmetric and positive definite and has the following structure

$$
\begin{aligned}
K= & \operatorname{diag}\left(K_{1}, K_{2 \_3}, K_{4 \_5}, K_{6 \_7}\right) \\
& K_{1} \in \mathbb{R}, K_{i}=K_{i}^{T} \in\left\{\mathbb{R}^{2 \times 2} \mid i=\left\{2 \_3,4 \_5,6 \_7\right\}\right\} .
\end{aligned}
$$

Furthermore $M(q) \in \mathbb{R}^{n \times n}$ is the mass matrix, $C(q, \dot{q}) \in$ $\mathbb{R}^{n \times n}$ the centrifugal and Coriolis matrix, and $g(q) \in \mathbb{R}^{n}$ the gravity vector of the rigid body model.

Due to the coupling of the joints through the differential gear (see Fig. 2), motor coordinates, denoted by the subscript $m$, have to be distinguished from the coordinates after the gearbox (or, on the link side), written without subscripts. For example, the motor position $\theta_{m}$ as well as the motor inertia $J_{m}$ are given in motor coordinates, while the joint torque $\tau$ is measured after the gear, in link coordinates. Due to the differential gears, the transformations between motor and link coordinates for positions and torques are given by

$$
\left\{\begin{array}{l}
\theta=T \theta_{m} \\
\tau_{m}=T^{T} \tau
\end{array}\right.
$$

with the transformation matrix e.g.

$$
T=\operatorname{diag}\left(1, T_{c}, T_{c}, T_{c}\right), \quad \forall T_{c}=\left[\begin{array}{rr}
0.5 & 0.5 \\
-0.5 & 0.5
\end{array}\right] .
$$

Finally, in order to facilitate the controller design and the stability analysis the following three properties are used
P.1: $\quad$ The mass matrix $M(q)$ is symmetric and positive definite $M(q)=M^{T}(q)$ and

$$
x^{T} M(q) x \geq 0, \quad \forall q, x \in \mathbb{R}^{n} .
$$

P.2: $\quad$ The matrix $\dot{M}(q)-2 C(q, \dot{q})$ is skew symmetric and

$$
x^{T}(\dot{M}(q)-2 C(q, \dot{q})) x=0, \quad \forall x, q, \dot{q} \in \mathbb{R}^{n} .
$$

P.3: For the friction moment $\tau_{f m}$ a dynamic friction model (LuGre friction model [10], [13]) is chosen as

$$
\tau_{f m}=\sigma_{0} z+\sigma_{1} \dot{z}+f_{v} \dot{\theta}_{m}
$$

with the inner dynamics of the friction

$$
\left\{\begin{array}{l}
\dot{z}=\dot{\theta}_{m}-\frac{\left|\dot{\theta}_{m}\right|}{h_{z}} \sigma_{0} z \\
h_{z}=f_{c}+\mu\left|\tau_{m}\right|
\end{array}\right.
$$

Therein, $\tau_{m}$ is the measured link torque in motor coordinates. $\sigma_{0}$ and $\sigma_{1}$ are the stiffness and damping coefficients of the LuGre friction model. $f_{c}, f_{v}$ and $\mu$ describe the Coulomb, viscous and load dependent coefficient of the friction moment, respectively.

For control design the dynamic equations (1) and (2) are transformed to the link coordinate system

$$
\begin{aligned}
u & =J \ddot{\theta}+\tau+\tau_{f} \\
\tau & =M(q) \ddot{q}+C(q, \dot{q}) \dot{q}+g(q)
\end{aligned}
$$

with the measured link torque

$$
\tau=K(\theta-q)
$$

by transformations (5) and

$$
\left\{\begin{array}{l}
J=T^{-T} J_{m} T^{-1} \\
u=T^{-T} u_{m} \\
\tau_{f}=T^{-T} \tau_{f m}
\end{array}\right.
$$

\section{DECOUPLING MIMO STATE FEEDBACK CONTROLLER}

In [8] a MIMO state feedback controller for the DLR medical robots has been designed through modal decomposition in order to deal with the high coupling between the robot joints. Therefor, using the torque controller

$$
u=J\left(\lambda_{J} K\right)^{-1} w+\left(I-J\left(\lambda_{J} K\right)^{-1}\right) \tau+\tau_{f},
$$

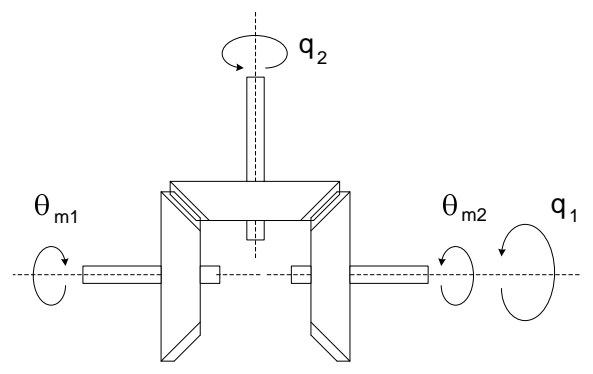

Fig. 2. Differential gear of the DLR medical robot. 
the motor dynamics can be transformed from the link coordinate system (8) to the modal coordinate system with $\lambda_{J}$ being a positive scalar and being chosen so $\left\{\left\|J\left(\lambda_{J} K\right)^{-1}\right\|\right.$ $-1\} \rightarrow \min$. The vector $w$ is a new control input and $I$ is the unit matrix. So one becomes the motor dynamics in the modal coordinate system

$$
w=\left(\lambda_{J} K\right) \ddot{\theta}+\tau
$$

\section{A. Control Law}

Let us call the desired link torque $\tau_{d}$. For a given desired link position $q_{d}$ and motor position $\theta_{d}$ the corresponding link torque is given by

$$
\tau_{d}=K\left(\theta_{d}-q_{d}\right)
$$

Then, the linear control law is presented by

$$
w=K_{P} e_{\theta}-K_{D} \dot{\theta}+K_{T} K^{-1} e_{\tau}-K_{S} K^{-1} \dot{\tau}+\tau_{d}
$$

with

$$
\left\{\begin{array}{l}
e_{\theta}=\theta_{d}-\theta \\
e_{\tau}=\tau_{d}-\tau .
\end{array}\right.
$$

In this control law the desired motor position and the desired link torque are chosen by

$$
\left\{\begin{array}{l}
\tau_{d}=g\left(q_{d}\right) \\
\theta_{d}=q_{d}+K^{-1} g\left(q_{d}\right) .
\end{array}\right.
$$

The control gain matrices $K_{P}, K_{D}, K_{T}$ and $K_{S}$ are positive definite and symmetric. Each control matrix can be represented in the following form [8]

$$
\begin{aligned}
& K_{C}=\left[\begin{array}{cccc}
K_{C_{1}} & \ldots & & 0 \\
& K_{C_{2-3}} & & \\
\vdots & & K_{C_{4-5}} & \vdots \\
0 & \ldots & & K_{C_{6-7}}
\end{array}\right] \\
& \forall C=\{P, D, T, S\}, \quad K_{C_{1}} \in \mathbb{R}, \\
& K_{C_{i}}=K_{C_{i}}^{T} \in\left\{\mathbb{R}^{2 \times 2} \mid i=\left\{2 \_3,4 \_5,6 \_7\right\}\right\}
\end{aligned}
$$

Furthermore, there exists a matrix $Q \in \mathbb{R}^{n \times n}$, such that the relationships

$$
\left\{\begin{array}{l}
K=Q Q^{T} \\
K_{P}=Q K_{P Q} Q^{T} \\
K_{D}=Q K_{D Q} Q^{T} \\
K_{T}=Q K_{T Q} Q^{T} \\
K_{S}=Q K_{S Q} Q^{T}
\end{array}\right.
$$

hold, with $K_{P Q}, K_{D Q}, K_{T Q}$ and $K_{S Q} \in \mathbb{R}^{n \times n}$ being positive definite and diagonal matrices.

\section{B. Robust Design of the Control Parameters}

Before the DLR medical robots are assembled, startup experiments are executed separately for each pair of coupled joints. The joint coupling of the coupled joints is dominant. So the control parameters can be designed independently for each pair of coupled joints (and single joints, respectively), which is considered as an 8th order system, according to a worst case position (e.g. maximal load). The pole placement for the control parameters is done by specifying a desired characteristic polynomial of the form

$$
\begin{array}{r}
\left(s+p_{1}\right)\left(s+p_{2}\right)\left(s^{2}+2 s \xi_{1} \omega_{1}+\omega_{1}^{2}\right)\left(s+p_{3}\right) \\
\left(s+p_{4}\right)\left(s^{2}+2 s \xi_{2} \omega_{2}+\omega_{2}^{2}\right) .
\end{array}
$$

Therefor, $p_{1}, p_{2}, p_{3}, p_{4}$ are real poles. Because of the low stiffness at the coupled joint structure of the DLR medical robots the damping coefficients are chosen $\xi_{1}=\xi_{2}=0.7$ such that the system is better damped.

The controller is used effectually at the DLR medical robots. It is robust and ensures the good dynamic behavior of the robots. But experiments have shown that for medical applications which need high accuracy, the controller must be improved.

\section{PROPOSED CONTROLLER}

In this section a new controller is developed. The proposed controller is a MIMO state feedback controller as well, but with the new desired motor position and the new desired link torque as well as their derivatives used for feedforward.

Let us define the position tracking errors of the link side by

$$
e_{q}=q_{d}-q
$$

So, together with (14) and (10), this leads to

$$
e_{\tau}=\tau_{d}-\tau=K\left(e_{\theta}-e_{q}\right)
$$

\section{A. Desired Torque for a State Feedback Controller}

The desired link torque $\tau_{d}$ is generated by using the rigid body dynamics [14]. However in this paper an additional control damping term is added in the control law to ensure the system stability

$$
\tau_{d}=M(q) \ddot{q}_{d}+C(q, \dot{q}) \dot{q}_{d}+g(q)+K_{q} \dot{e}_{q}
$$

where the matrix $K_{q}$ is positive definite and diagonal.

Using (23) and (9) we obtain the closed-loop of the link dynamics

$$
\begin{aligned}
e_{\tau} & =\tau_{d}-\tau \\
& =M(q) \ddot{e}_{q}+C(q, \dot{q}) \dot{e}_{q}+K_{q} \dot{e}_{q} .
\end{aligned}
$$

\section{B. Full State Feedback Controller with Feedforward}

To ensure that the link position $q$ converges to the designed link position $q_{d}$, a computed torque like state feedback control law is chosen as

$$
\begin{aligned}
w= & \left(\lambda_{J} K\right) \ddot{\theta}_{d}+K_{P} e_{\theta}+K_{D} \dot{e}_{\theta} \\
& +K_{T} K^{-1} e_{\tau}+K_{S} K^{-1} \dot{e}_{\tau}+\tau_{d}
\end{aligned}
$$

with $K_{P}, K_{D}, K_{T}$ and $K_{S}$ being positive definite and symmetric matrices. These control matrices have the same characteristics as the control matrices (19) in Sec. III.

Then, substituting (25) into (13), we get the closed-loop motor dynamics

$$
\begin{aligned}
\left(\lambda_{J} K\right) & \ddot{e}_{\theta}+K_{P} e_{\theta}+K_{D} \dot{e}_{\theta} \\
& +\left(K_{T}+K\right) K^{-1} e_{\tau}+K_{S} K^{-1} \dot{e}_{\tau}=0 .
\end{aligned}
$$




\section{Stability Analysis}

For stability analysis the following Lyapunov function candidate was chosen

$$
\begin{aligned}
V= & \frac{1}{2} \dot{e}_{\theta}^{T} K\left(K_{T}+K\right)^{-1}\left(\lambda_{J} K\right) \dot{e}_{\theta} \\
& +\frac{1}{2} \dot{e}_{q}^{T} M(q) \dot{e}_{q}+\frac{1}{2} e_{\theta}^{T} K\left(K_{T}+K\right)^{-1} K_{P} e_{\theta} \\
& +\frac{1}{2}\left(e_{\theta}-e_{q}\right)^{T} K\left(e_{\theta}-e_{q}\right) .
\end{aligned}
$$

In accordance with the condition (19) of the control parameters, one can determine the matrices

$$
\left\{\begin{array}{l}
K\left(K_{T}+K\right)^{-1}\left(\lambda_{J} K\right)=\lambda_{J} Q\left(K_{T Q}+I\right)^{-1} Q^{T} \\
K\left(K_{T}+K\right)^{-1} K_{P}=Q\left(K_{T Q}+I\right)^{-1} K_{P Q} Q^{T}
\end{array}\right.
$$

Because the matrices $K_{T Q}$ and $K_{P Q} \in \mathbb{R}^{n \times n}$ are positive definite and diagonal, $K\left(K_{T}+K\right)^{-1}\left(\lambda_{J} K\right)$ and $K\left(K_{T}+\right.$ $K)^{-1} K_{P}$ are symmetric and positive definite as well. So the function $V$ is positive definite.

The derivative of the function $V$ along the trajectory, using equations (24) and (26), leads to

$$
\begin{aligned}
\dot{V}= & \dot{e}_{\theta}^{T} K\left(K_{T}+K\right)^{-1}\left(\lambda_{J} K\right) \ddot{e}_{\theta}+\dot{e}_{q}^{T} M(q) \ddot{e}_{q} \\
& +\frac{1}{2} \dot{e}_{q}^{T} \dot{M}(q) \dot{e}_{q}+\dot{e}_{\theta}^{T} K\left(K_{T}+K\right)^{-1} K_{P} e_{\theta} \\
& +\left(\dot{e}_{\theta}-\dot{e}_{q}\right)^{T} K\left(e_{\theta}-e_{q}\right) \\
= & -\dot{e}_{\theta}^{T} K\left(K_{T}+K\right)^{-1}\left(K_{D}+K_{S}\right) \dot{e}_{\theta} \\
& +\dot{e}_{\theta}^{T} K\left(K_{T}+K\right)^{-1} K_{S} \dot{e}_{q}-\dot{e}_{q}^{T} K_{q} \dot{e}_{q} \\
:= & -\left[\begin{array}{ll}
\dot{e}_{\theta}^{T} & \dot{e}_{q}^{T}
\end{array}\right] \mathrm{H}\left[\begin{array}{c}
\dot{e}_{\theta} \\
\dot{e}_{q}
\end{array}\right]
\end{aligned}
$$

with symmetric Hessian matrix ${ }^{1}$

$$
H=\left[\begin{array}{cc}
K\left(K_{T}+K\right)^{-1}\left(K_{D}+K_{S}\right) & -\frac{1}{2} K\left(K_{T}+K\right)^{-1} K_{S} \\
-\frac{1}{2} K\left(K_{T}+K\right)^{-1} K_{S} & K_{q}
\end{array}\right] .
$$

The function $\dot{V}$ is negative definite when the Hessian matrix $H$ is positive definite. This leads to the condition for sub-matrices $H_{22}>H_{12} H_{11}^{-1} H_{21}$ or

$$
K_{q}>\frac{1}{4} K\left(K_{T}+K\right)^{-1} K_{S}\left(K_{D}+K_{S}\right)^{-1} K_{S}
$$

Therefore, the functions $V>0$ and $\dot{V} \leq 0$. This implies that $V(t) \leq V(0)$, and therefor, that $e_{\theta}, \dot{e}_{\theta}, e_{q}$ and $\dot{e}_{q}$ are bounded. Because the derivative of $\dot{V}$ (using equations (24) and (26)) is bounded as well, according to Barbalat's lemma [14] the function $\dot{V}$ is uniformly continuous, or $\dot{V} \rightarrow 0$ as $t \rightarrow \infty$. That leads to $\left\{\lim _{t \rightarrow \infty} \dot{e}_{\theta}=0, \lim _{t \rightarrow \infty} \dot{e}_{q}=\right.$ $0\}$, hence, $\left\{\lim _{t \rightarrow \infty} \ddot{e}_{\theta}=0, \lim _{t \rightarrow \infty} \ddot{e}_{q}=0\right\}$. From the closed-loop of the dynamics (24), (26), it is shown that that the position errors converge to zero $\left\{\lim _{t \rightarrow \infty} e_{\theta}=\right.$ $\left.0, \lim _{t \rightarrow \infty} e_{q}=0\right\}$, or $\left\{\theta \rightarrow \theta_{d}, q \rightarrow q_{d}\right\}$. The system is globally asymptotically stable.

\footnotetext{
${ }^{1}$ The matrices $K\left(K_{T}+K\right)^{-1}\left(K_{D}+K_{S}\right)=Q\left(K_{T Q}+I\right)^{-1}\left(K_{D Q}+\right.$ $\left.K_{S Q}\right) Q^{T}$ and $K\left(K_{T}+K\right)^{-1} K_{S}=Q\left(K_{T Q}+I\right)^{-1} K_{S Q} Q^{T}$ are symmetric and positive definite according to the condition (19)
}

\section{EXPERIMENTS}

In this section the results of experiments with the controllers of Sec. III (the state feedback controller without feedforward terms, with model-based friction compensation) and Sec. IV (the state feedback controller with feedforward terms, with model-based friction compensation) are presented with the DLR medical robot. Furthermore, it is shown that the accuracy of the robots can be improved even more if the new control structure with feedforward terms is combined with the disturbance observer of [12] to compensate the friction effects. So the experimental results of this combination (the state feedback controller with feedforward terms, with observer-based friction compensation) are reported as well in this section. The experiment results of the coupled joint 2-3, for which the load is dominant, are shown to validate the control structures. The tables I, II and III show the parameters of the coupled joint 2-3, as an example.

TABLE I

PARAMETERS OF THE COUPLED JOINT 2-3.

\begin{tabular}{|l|l|}
\hline Motor inertial $J_{m}\left(\mathrm{kg.m} \mathrm{m}^{2}\right)$ & {$[1.26813,1.26813]$} \\
\hline Stiffness matrix $K(\mathrm{Nm} / \mathrm{rad})$ & {$\left[\begin{array}{rr}2700.0, & -101.10 \\
-101.10, & 3035.6\end{array}\right]$} \\
\hline Damping matrix $D(\mathrm{Nm} . \mathrm{s} / \mathrm{rad})$ & {$\left[\begin{array}{rr}5.06, & -0.59 \\
-0.59, & 7.68\end{array}\right]$} \\
\hline
\end{tabular}

TABLE II

FRICTION PARAMETERS OF THE MOTORS 2-3.

\begin{tabular}{|c|c|c|c|c|c|}
\hline Motor & $\begin{array}{c}f_{c} \\
(\mathrm{Nm})\end{array}$ & $f_{l}$ & $\begin{array}{c}f_{v} \\
(\mathrm{Nm} . \mathrm{s} / \mathrm{rad})\end{array}$ & $\begin{array}{c}\sigma_{0} \\
(\mathrm{Nm} / \mathrm{rad})\end{array}$ & $\begin{array}{c}\sigma_{1} \\
(\mathrm{Nm} . \mathrm{s} / \mathrm{rad})\end{array}$ \\
\hline 2 & 10.51 & 0.1263 & 14.56 & 5033.38 & 317.66 \\
\hline 3 & 10.36 & 0.1432 & 15.48 & 5423.55 & 286.50 \\
\hline
\end{tabular}

TABLE III

MIMO STATE FEEDBACK CONTROLLER PARAMETERS OF THE COUPLED JOINT 2-3.

\begin{tabular}{|c|c|}
\hline$K_{P}$ & $K_{D}$ \\
\hline$\left[\begin{array}{cc}7606.33, & 144.33 \\
144.33, & 7120.02\end{array}\right]$ & {$\left[\begin{array}{cc}465.08, & 6.61 \\
6.61, & 442.70\end{array}\right]$} \\
\hline \hline$K_{T} K^{-1}$ & $K_{S} K^{-1}$ \\
\hline$\left[\begin{array}{cc}6.04824, & 0.08149 \\
0.08143, & 5.77639\end{array}\right]$ & {$\left[\begin{array}{cc}0.004742, & -0.006841 \\
-0.006837, & 0.027564\end{array}\right]$} \\
\hline \hline$K_{q}$ & {$\left[\begin{array}{c}0.00033\end{array}\right]$} \\
\hline$\left[\begin{array}{cc}2.0, & 0.0 \\
0.0, & 2.0\end{array}\right]$ & $\lambda_{J}$ \\
\hline
\end{tabular}

In the first experiment, the robot follows a periodic trajectory (see Fig. 3) in order to show the behavior in terms of tracking errors. The motor position errors of both controllers according to Sec. III and Sec. IV are shown in Fig. 4. It can be seen from the tracking errors that the state feedback controller with new feedforward terms is considerably superior in performance with respect to the controller without feedforward terms. The dynamic behavior of the robot in those two cases is compared by the measured link torque in Fig. 5. At the reversal points of the trajectory 

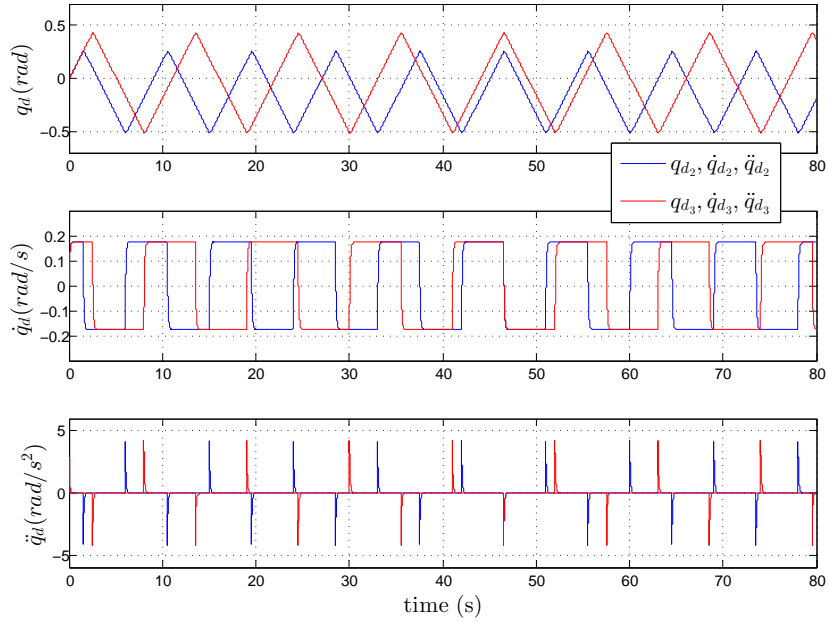

Fig. 3. Desired link position and velocity, periodic trajectory.

it can be seen that the new feedforward terms inhibit hardly damped oscillations by the link torques, which characterize the state feedback controller without feedforward terms in Sec. III.

Furthermore, the state feedback controller with feedforward terms can be combined with observer-based friction compensation [12] instead of model-based friction compensation to achieve a higher accuracy (see Fig. 6). In case of the state feedback controller without feedforward terms the experiments show that a combination with observer-based friction compensation improves the tracking accuracy of the robots not as much.

In the second experiment, a point to point trajectory (see Fig. 7) is chosen in order to show the position tracking accuracy of the robots. The best performance is clearly obtained by the new control structure using the feedforward (see Fig. 8). Fig. 9 shows the improvement of the position accuracy, when the state feedback controller with feedforward terms uses observer-based friction compensation instead of model-based friction compensation.

However, in both experiments some steady state error can be seen because of the coarsely modeled friction torque and rigid body dynamics.

It can be concluded that the new controller with the feedforward considerably contributes to the reduction of the positioning errors.

\section{CONCLUSIONS}

In this paper we have proposed a new state feedback control scheme with the new desired motor position and the new desired link torque as well as their derivatives as a feedforward that can be used in order to enhance the robot accuracy. Finally, global asymptotic stability of the controller has been proven. Experimental results validate the approach for the DLR medical robot.

\section{REFERENCES}

[1] T. Ortmaier, H. Weiss, U. Hagn, M. Grebenstein, M. Nickl, A. AlbuSchäffer, C. Ott, S. Jörg, R. Konietschke, L. Le-Tien, and G. Hirzinger. A hands-on-robot for accurate placement of pedicle screws. IEEE
International Conference on Robotics and Automation, pages 41794186, 2006.

[2] U. Hagn, M. Nickl, S. Jörg, G. Passig, T. Bahls, A. Nothhelfer F. Hacker, L. Le-Tien, A. Albu-Schäffer, R. Konietschke, M. Grebenstein, R. Warpup, R. Haslinger, M. Frommberger, and G. Hirzinger. The DLR MIRO: A versatile lightweight robot for surgical applications. Industrial Robot: An International Journal, pages 324 - 336, 2008.

[3] P. Tomei. A simple PD controller for robots with elastic joints. IEEE Transaction on Robotics and Automation, pages 1208-1213, 1991.

[4] A. Albu-Schäffer and G. Hirzinger. A globally stable state-feedback controller for flexible joint robots. Journal of Advanced Robotics, pages 799-814, 2001.

[5] A. De Luca and P. Lucibello. A general algorithm for dynamic feedback linearization of robots with elastic joints. IEEE International Conference on Robotics and Automation, pages 504-510, 1998.

[6] B. Brogliato, R. Ortega, and R. Lozano. Global tracking controllers for flexible-joint manipulators: a comparative study. Automatica, 31:941956, 1995.

[7] L. Tian and A.A. Goldenberg. Robust adaptive control of flexible joint robots with joint torque feedback. IEEE International Conference on Robotics and Automation, pages 1229-1234, 1995.

[8] L. Le-Tien, A.Albu-Schäffer, and G. Hirzinger. MIMO state feedback controller for a flexible joint robot with strong joint coupling. IEEE International Conference on Robotics and Automation, pages 3824 3830, 2007.

[9] B. S. R. Armstrong. Dynamics for robot control: Friction modeling and ensuring excitation during parameter identification. Dissertation, Stanford University, 1988.

[10] C. C. de Wit, H. Olsson, K. J. Astron, and P. Linschinsky. A New Model for Control of Systems with Friction. IEEE Transaction on Automatic Control, 40:419-425, 1994.

[11] L. Le-Tien and A.Albu-Schäffer. Adaptive Friction Compensation in Trajectory Tracking Control of DLR Medical Robots with Elastic Joints. IEEE/RSJ 2012 International Conference on Intelligent Robots and Systems, 2012.

[12] L. Le-Tien, A.Albu-Schäffer, A. De Luca, and G. Hirzinger. Friction observer and compensation for control of robots with joint torque measurement. IEEE/RSJ 2008 International Conference on Intelligent Robots and Systems, pages 3789 - 3795, 2008.

[13] L. Le-Tien. Ansätze zur entkoppelten Regelung von mechanisch gekoppelten Doppelgelenken eines DLR - Medizinroboters. Dissertation, Technische Universität Dresden, 2010.

[14] J-J. E. Slotine and W. Li. Applied Nonlinear Control. Prentice-Hall International, 1991
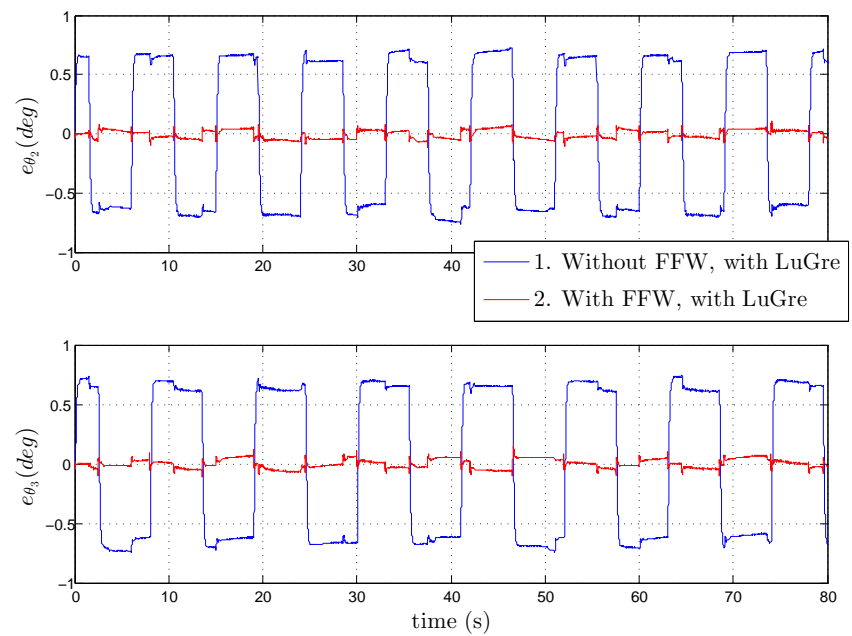

Fig. 4. Tracking motor position errors of the MIMO state feedback control scheme during the periodic trajectory: 1) without feedforward and with model-based friction compensation 2) with feedforward and with modelbased friction compensation. 

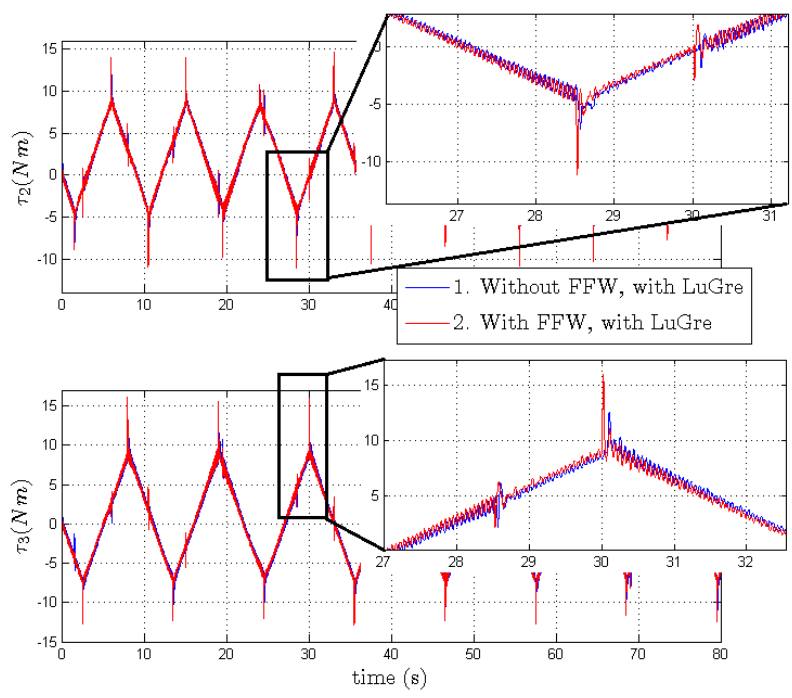

Fig. 5. Measured link torque of the MIMO state feedback control scheme during the periodic trajectory: 1) without feedforward and with model-based friction compensation 2) with feedforward and with model-based friction compensation.
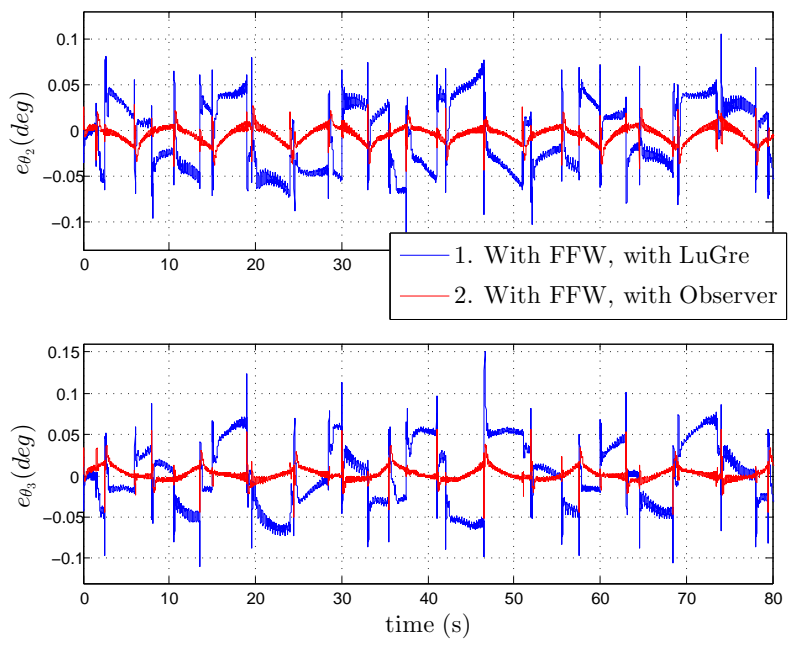

Fig. 6. Tracking motor position errors of the MIMO state feedback control scheme during the periodic trajectory: 1) with feedforward and with modelbased friction compensation 2) with feedforward and with observer-based friction compensation.
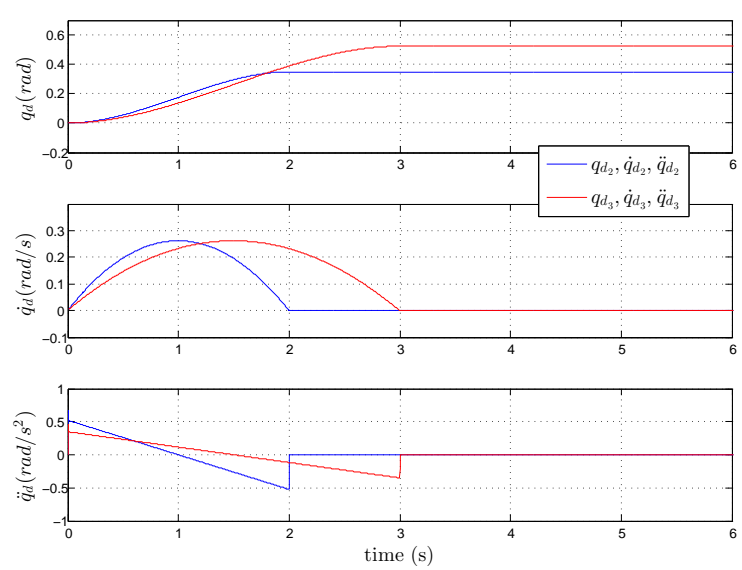

Fig. 7. Desired link position and velocity, point to point trajectory.
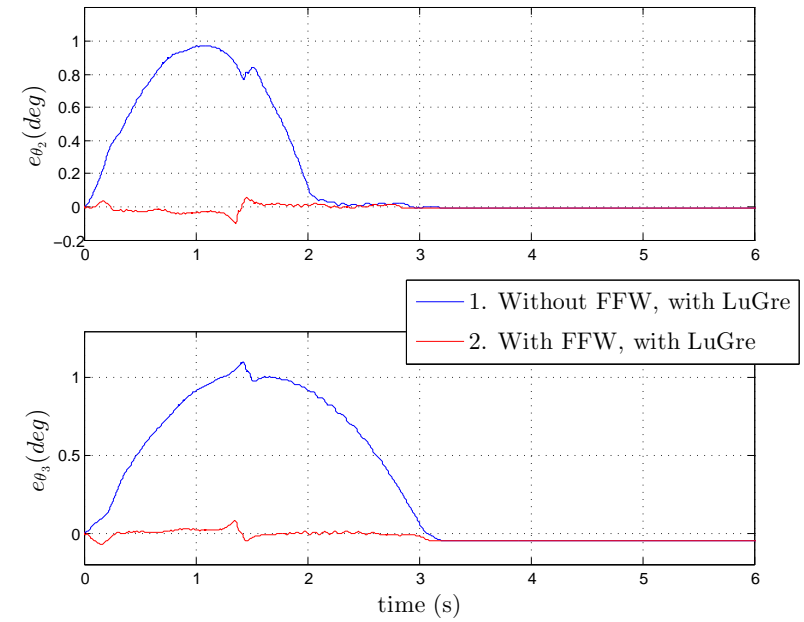

Fig. 8. Tracking motor position errors of the MIMO state feedback control scheme during the point to point trajectory: 1) without feedforward and with model-based friction compensation 2) with feedforward and with modelbased friction compensation.
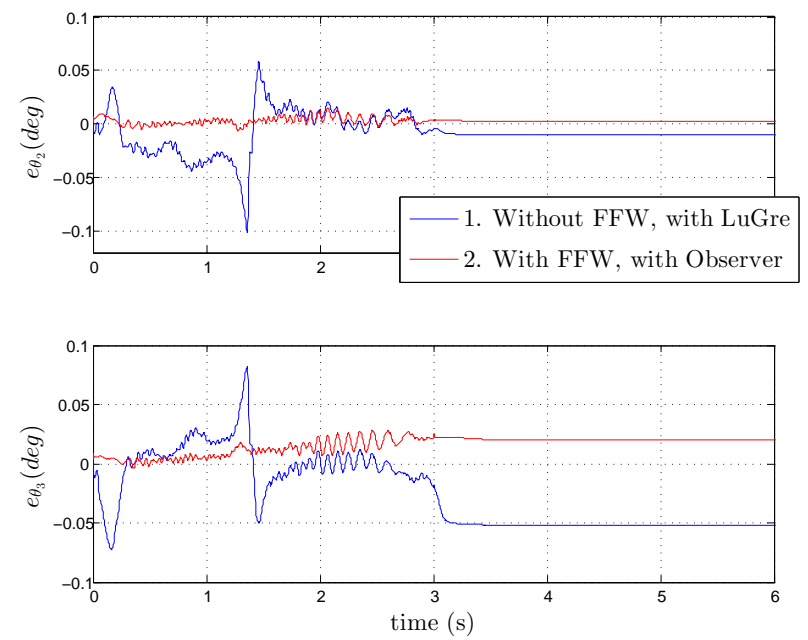

Fig. 9. Tracking link position errors of the new MIMO state feedback control scheme during the point to point trajectory: 1) with feedforward and with model-based friction compensation 2) with feedforward and with observer-based friction compensation. 